\section{Proteasome Subunit LMP2/ $\beta$ li As a Biomarker for Human Uterine Mesenchymal Tumors}

\section{Journal of Oncobiomarkers}

\author{
Takuma Hayashi $i^{1,12,13 *}$, Akiko Horiuchi ${ }^{2}$, Kenji \\ Sano $^{3}$, Gal Gur ${ }^{4,13}$, Hiroyuki Aburatani ${ }^{5}$, Osamu \\ Ishiko ${ }^{6}$, Nobuo Yaegashi', Tanri Shiozawa ${ }^{8}$, Yae \\ Kanai $^{9,14}$, Dorit Zharhary ${ }^{4,13}$, Susumu Tonegawa ${ }^{10}$ \\ and Ikuo Konishi'
}

${ }^{1}$ Dept. of Immunology and Infectious Disease, Shinshu University, School of Medicine, Matsumoto, Nagano 390-8621, Japan ${ }^{2}$ Horiuchi Ladies Clinic, Matsumoto, Nagano 390-0821, Japan ${ }^{3}$ Department of Laboratory Medicine, Shinshu University Hospital, Matsumoto, Nagano 390-8621, Japan

${ }^{4}$ Sigma-Aldrich Israel Ltd., Rehovot 76100, Israel

${ }^{5}$ The Cancer System Laboratory, Research Center for Advanced Science and Technology, The University of Tokyo, Meguro, Tokyo 153-9804 Japan

${ }^{6}$ Department of Obstetrics and Gynecology, Osaka City University Graduate School of Medicine, Osaka, Osaka 545-8585 Japan ${ }^{7}$ Department of Obstetrics and Gynecology, Tohoku University Graduate School of Medicine, Sendai, Miyagi 980-8574 Japan ${ }^{8}$ Department of Obstetrics and Gynecology, Shinshu University School of Medicine, Matsumoto, Nagano 390-8621, Japan ${ }^{9}$ Pathology Division, National Cancer Center Research Institute, Chuoku, Tokyo 104-0045, Japan

${ }^{10}$ Picower Institution and Department of Biology, Massachusetts Institute of Technology, Cambridge, MA 02139-4307 USA

${ }^{11}$ Department of Obstetrics and Gynecology, Kyoto University Graduate School of Medicine, Kyoto, Kyoto 606-8507, Japan

${ }^{12}$ Promoting Business using Advanced Technology, Japan Science and Technology Agency (JST), Chiyoda, Tokyo 102-8666, Japan ${ }^{13}$ SIGMA-Aldrich Collaboration Laboratory

${ }^{14}$ The International Human Epigenome Consortium (IHEC) and CREST, Japan Science and Technology Agency (JST), Chiyoda, Tokyo 102-8666, Japan

\section{Address for Correspondence}

Takuma Hayashi, Department of Immunology and Infectious Disease, Shinshu University Graduate School of Medicine, 3-1-1, Asahi, Matsumoto, Nagano 390-8621, Japan, Tel: 81-263-37-2611; Fax: 81263-37-2613; E-mail: yoyoyo224@hotmail.com

Copyright: (c) 2013 Hayashi T, et al. This is an open access article distributed under the Creative Commons Attribution License, which permits unrestricted use, distribution, and reproduction in any medium, provided the original work is properly cited.

LMP2: low-molecular mass polypeptide 2, Ut-LMS: uterine leiomyosarcoma, LMA: leiomyoma, SMT: smooth muscle tumor, JAK: Janus kinase, IFN- $\gamma$ : interferon- $\gamma$, MECL-1: multicatalytic endopeptidase complex-like.

\section{Introduction}

Proteins play crucial roles in all biological processes and, the finely tuned equilibrium between their synthesis and degradation regulates cellular homeostasis. Controlling the quality of proteome informational content is essential for cell survival and function. After their initial synthesis, membrane and secretory proteins are modified, folded, and assembled in the endoplasmic reticulum, whereas other proteins are synthesized and processed in the cytosol. Cells have different protein quality control systems, the molecular chaperones, which assist protein folding and stabilization, and the ubiquitin-proteasome system (UPS) and lysosomes, which degrade proteins. UPS and lysosomes are generally assumed to be regulated independently and serve distinct functions. Proteasomes, which are located in the nucleus and cytoplasm, have a hollow barrel-shaped multiprotein structure of $2.5 \mathrm{MDa}$ and are comprised of a core particle (20S proteasome) flanked on the both sides by a regulatory particle (19S proteasome) [1,2]. The functions of the $19 \mathrm{~S}$ proteasome include the recognition of ubiquitinated proteins, denaturing of these proteins, de-ubiquitination, which allows ubiquitin molecules to be recycled, and the delivery of target proteins to the $20 \mathrm{~S}$ proteasome [3].
Submission: 14 November 2013

Accepted: 04 December 2013

Published: 13 December 2013

Reviewed \& Approved by: Dr. Youming Xie

Associate Professor, Department of Oncology \& Karmanos Cancer

Institute, Wayne State University School of Medicine, USA

The $20 \mathrm{~S}$ proteasome consists of four rings of seven member proteins, each of which are joined stuck one on the other. The two peripheral rings are identical and called $\alpha$-rings (with subunits $\alpha 1$ to $\alpha 7$ ). The two central rings are also identical and called $\beta$-rings (with subunits $\beta 1$ to $\beta 7$ ). The proteasome possesses three enzymatic activities; trypsinlike (cleavage of post-basic residues) activity, a chymotrypsin-like (cleavage of post-hydrophobic residues) activity, and a post-glutamyl (cleavage of caspase-like or post-acidic residues) activity that reside in subunits $\beta 1, \beta 2$, and $\beta 5$ respectively and degrade target proteins producing fragments of 4 to 14 amino acids [4]. The proteasome is a multiprotein complex that regulates the stability of hundreds of cellular proteins and is thus, implicated in virtually all cellular functions, including cell proliferation, apoptosis, angiogenesis, and motility, which are of particular importance for tumorigenesis. 
Citation: Hayashi T, Horiuchi A, Sano K, Gur G, Aburatani H, et al. Proteasome Subunit LMP2/b1i As a Biomarker for Human Uterine Mesenchymal Tumors. J Oncobiomarkers. 2013;1(2): 6.

Alternative $\beta$-forms of low molecular weight protein (LMP)2/ $\beta 1 \mathrm{i}$ are expressed in the myometrium following exposure to proinflammatory signals such as cytokines, especially IFN $-\gamma$. Uterine leiomyosarcoma (Ut-LMS) was shown to occurred in female LMP2/ $\beta 1$ i-null mice at 6 months or older, and its incidence at 14 months approximately $40 \%$ [5,6]. Human uterine mesenchymal tumors, i.e. smooth muscle tumors (SMTs), which develop in the myometrium, have traditionally been divided into benign leiomyoma (LMA) and malignant Ut-LMS based on cytological atypia, mitotic activity, and other criteria. Ut-LMS is relatively rare, having an estimated annual incidence of 0.64 per 100,000 women [7]. Surgical intervention is virtually the only means of treatment because Ut-LMS is resistant to chemotherapy and radiotherapy [8-10]. The prognosis of patients with Ut-LMS is poor, and the five-year survival rate is approximately $35 \%$. However, the development of an efficient adjuvant therapy is expected to improve the prognosis of patients with Ut-LMS. Uterine LMA may occur in as $70 \% \sim 80 \%$ of women by the age of 50 years [11]. However, difficulties are associated with distinguishing uterine LMA from Ut-LMS, and a diagnosis generally requires surgery and cytoscopy [12]. Diagnostic categories for uterine SMTs and morphological criteria are used to assign cases $[13,14]$. The nonstandard subtypes of uterine SMTs such as the epithelioid and myxoid types are classified differently using these features; therefore establishing a diagnostic method to identify non-standard smooth muscle differentiation is important $[13,14]$.

High estrogen levels are considered to significantly influence the development of tumors in the uterine body [15-17]. The molecular mechanisms by which uterine LMA and Ut-LMS develop have not yet been elucidated, however, tumors that develop in the myometrium gradually increase in size due to the influence of the female hormone and, estrogen, and generate tumors. No correlation between the development of Ut-LMS and hormonal conditions, and no obvious risk factors have been identified. Although cases accompanied by hypocalcaemia or eosinophilia have been reported, neither clinical abnormality is an initial risk factor for Ut-LMS. Determining the malignant potential of smooth muscle neoplasms also represents a significant diagnostic conundrum with important therapeutic ramifications. However, the genetic changes underlying the neoplastic transformation of uterine smooth muscle cells have not been fully characterized. Moreover, diagnostic biomarkers that can distinguish between Ut-LMS and uterine mesenchymal tumors including LMA have yet to be established. The protein homogeny of the proteasome $\beta$-subunit, LMP2/ $\beta 1 \mathrm{i}$ in humans and mice is $88.1 \%$, and further studies on human and mouse uterine tissues revealed the defective expression of LMP2/ß1i in human Ut-LMS. The identification of a risk factor and/or biological candidate(s) associated with the development of Ut-LMS, i.e. LMP $2 / \beta 1$ i, may significantly contribute to the development of preventive and therapeutic treatments.

\section{Defective LMP2/ $\beta 1$ i expression in human Ut-LMS}

Cytoplasmic proteins are mostly degraded by a protease complex, which has many substrates consisting of twenty-eight 20 to $30 \mathrm{kDa}$ subunits, referred to as the $20 \mathrm{~S}$ proteasome, and is located in the nucleus and cytoplasm [18,19]. Proteasomal degradation plays an essential role in for many cellular processes, including the cell cycle, regulation of gene expression, and immunological function. The abnormal expression of proteasome subunits has been correlated with tumor initiation and progression $[20,21]$. IFN- $\gamma$ was shown to induce the expression of large numbers of responsive genes encoding proteasome subunits, i.e., LMP $2 / \beta 1 \mathrm{i}, \mathrm{LMP} / \beta 5 \mathrm{i}$, and LMP10/multicatalytic endopeptidase complex-like (MECL)-1/ $\beta 2 \mathrm{i}$ [22]. The individual expression of the LMP $2 / \beta 1 \mathrm{i}, \mathrm{LMP} 7 / \beta 5 \mathrm{i}$, and LMP10(MECL-1)/ $\beta 2 \mathrm{i}$ subunits is believed to contribute to the initiation and development of disorders. A recent study revealed the unique role of LMP7/ $35 \mathrm{i}$ in controlling pathogenic immune responses and provided a therapeutic rationale for targeting LMP7/ $\beta 5 \mathrm{i}$ in autoimmune disorders, especially rheumatoid arthritis (RA) [23]. The selective inhibition of LMP7/ $\beta 5$ i blocked the production of interleukin-23 (IL-23) by activated monocytes and also IFN- $\gamma$ and IL-2 by $\mathrm{T}$ cells. In mouse models of RA, LMP7/ $/ 35$ i-inhibitory treatments were shown to reverse the signs of disease and resulted in reductions in cellular infiltration, cytokine production, and autoantibody levels. Recent studies have suggested that LMP2/ $\beta 1 \mathrm{i}$ is necessary for tumor surveillance and may play a tissue-specific role in protection against spontaneous uterus neoplasms $[5,24]$. The presentation of antigenic peptides by MHC class I molecules is important for tumor rejection by CTLs.

Such antigenic peptides are generated as a result of the degradation of intracellular proteins by the proteasome pathway, a process that is influenced by the LMP2/ $\beta$ 1i subunit of the proteasome complex (Figure 1a). Homozygous mice deficient in LMP2/ $\beta 1$ i exhibit tissueand substrate-dependent abnormalities in the biological functions of the proteasome [5,24]. Ut-LMS has been reported in female LMP2/ $\beta 1$ i-null mice at 6 months or older, and its incidence at 14 months was approximately $40 \%$ [5,6] (Figure $1 \mathrm{~b}$ ). The disease prevalence in mice is similar to that of human Ut-LMS, which occurs after menopause. Pathological studies on LMP2/ $\beta 1$ i-null uterine tumors revealed the characteristic abnormalities of human Ut-LMS [5]. These tumors lacked lymphoid infiltrates, a sign of immune recognition, and consisted of uniformly elongated smooth muscle cells arranged into bundles. The nuclei of these tumor cells varied in size and shape; furthermore, mitosis was frequent. In contrast, the myometrium cells of C57BL/6 mice were normal in appearance [5]. Whereas relatively few ki-67-positive cells, which are proliferating cells, were observed in the basal cell layer of the normal myometrium, most basal cells in LMP2/ $\beta 1$ i-null mice vividly expressed ki-67 [5] (Figure 1b). This immunological staining revealed the abnormal proliferation of LMP2/ $\beta 1$ i-lacking cells in the basal layer. LMP2/ $\beta 1$ i-null mice with Ut-LMS lose a marked amount of weight and die by 14 months. They may also exhibit skeletal muscle metastasis from Ut-LMS [25]. Therefore, LMP2/ $/ 31$ i-null mice with Ut-LMS are more likely to die as a result of tumor growth and metastasis. It is generally not easy to distinguish uterine LMA from Ut-LMS in humans, however, in mice, because of the characteristic pathological findings, including significant weight loss, and possible skeletal muscle metastasis, a tumor that develops in the uterus of an LMP2/ $\beta 1$ i-null mouse can be considered as malignant, i.e., Ut-LMS [5,6] (Figure 1b).

To examine whether the biological significance of LMP2/ $\beta 1$ i to uterine sarcomagenesis observed in mice applies to other species, the mouse LMP2/ $\beta 1$ i protein sequence was compared with all protein sequences in euteleostomi using BLAST protein. Pairwise Alignment Scores, based on the homogeny of mouse LMP2/ $\beta 1$ i between other species, were 66.5 95\% (Figure 2). The protein homogeny of LMP2/ $\beta 1 \mathrm{i}$ in humans and mice was found to be $88.1 \%$ (Table 1 (included as supplementary data)). Furthermore, immunohistochemistry (IHC) revealed that the ability of human Ut-LMS tissue to induce LMP2/ $\beta 1 \mathrm{i}$ expression was markedly weaker than that of LMA or the normal 
Citation: Hayashi T, Horiuchi A, Sano K, Gur G, Aburatani H, et al. Proteasome Subunit LMP2/b1i As a Biomarker for Human Uterine Mesenchymal Tumors. J Oncobiomarkers. 2013;1(2): 6.
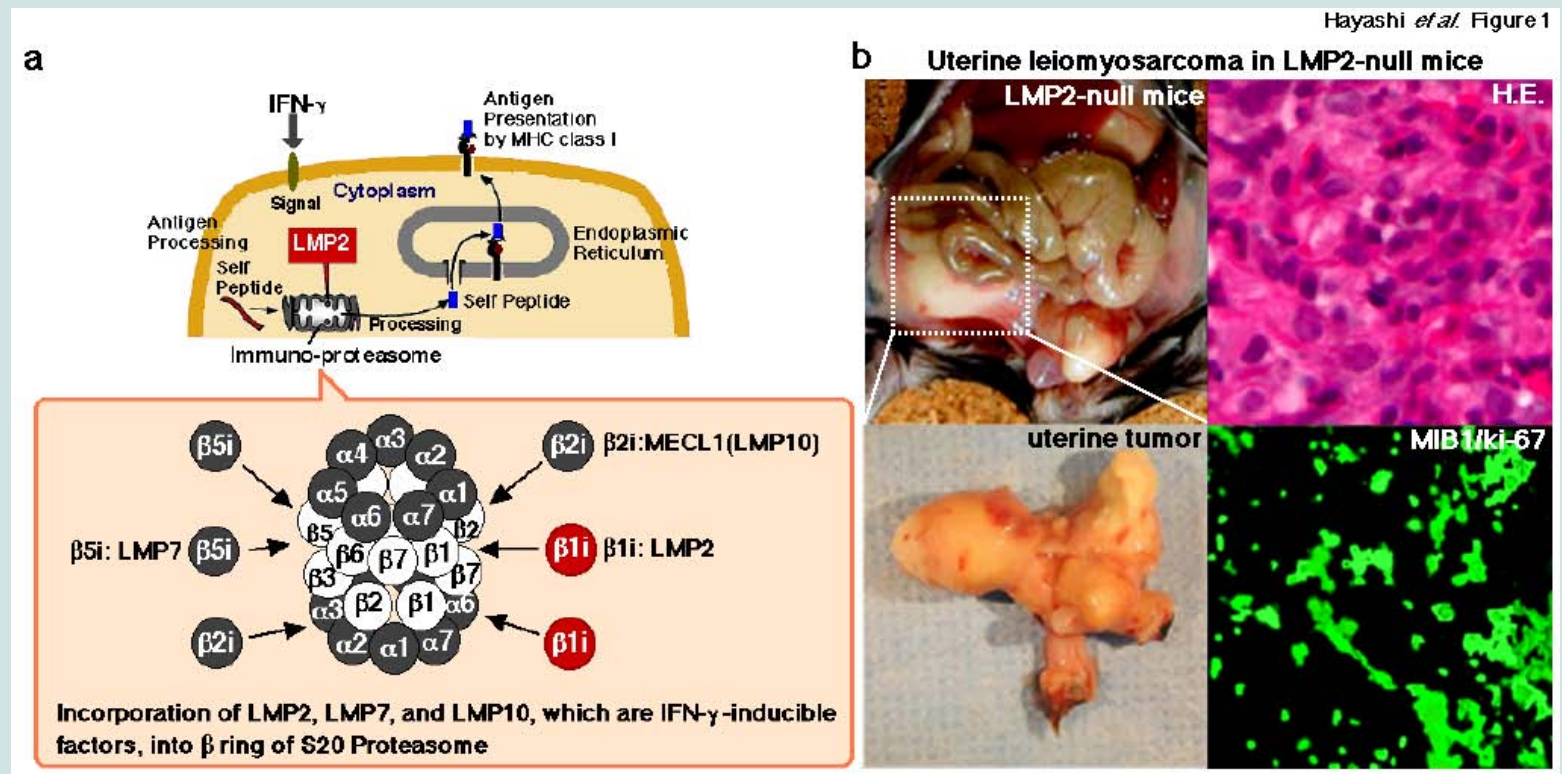

Figure 1: a. Mediation of the proteasomal degradation pathway to antigen presentation by MHC class I. The immuno-proteasomal degradation pathway is essential for antigen presentation by MHC class I. The defective expression of LMP2 results in tissue-and substrate-dependent abnormalities in immuno-proteasomal functions. Therefore, an impaired proteasome may promote the initial development of disease including tumorigenesis.

b. Homozygous mice deficient in LMP2/ $\beta 1$, an interferon (IFN)- $y$-inducible factor, show tissue-and substrate-dependent abnormalities in the biological functions of the proteasome $[5,27]$. Ut-LMS reportedly occurred in female LMP2/B1i-null mice at 6 months or older, and its incidence at 14 months was approximately $40 \%$ $[5,27]$

\section{Genes}

Genes identified as putative homologs of one another during the construction of HomoloG ene.

PSMB9, H. sapiens

proteasome (prosome, macropain) subunit, beta type, 9 (large multifunctional peptidase 2)

PSMB9, P.troglodytes

proteasome (prosome, macropain) subunit, beta

type, 9 (large multifunctional peptidase 2)

PSMB9, M. mulatta

proteasome (prosome, macropain) subunit, beta

type, 9 (large multifunctional peptidase 2)

PSMB9, C.lupus

proteasome (prosome, macropain) subunit, beta

type, 9 (large multifunctional peptidase 2)

PSMB9, B.taurus

proteasome (prosome, macropain) subunit, beta

type, 9 (large multifunctional peptidase 2)

Psmb9, M.musculus

proteasome (prosome, macropain) subunit, beta

type 9 (large multifunctional peptidase 2)

Psmb9, R.norvegicus

proteasome (prosome, macropain) subunit, beta type 9 (large multifunctional peptidase 2)

psmb9a, D.rerio

proteasome (prosome, macropain) subunit, beta type, 9a

\section{Proteins}

Proteins used in sequence comparisons and their conserved domain architectures

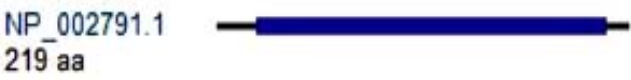

XP 001167429.1

$21 \overline{8}$ aa

XP 001108087.2

$21 \overline{9}$ aa

NP 001041551.1

209 aa

NP_001029560.1

$219^{-}$aa

NP_038613.1

219 aa

NP_036840.2

219 aa

NP 571466.1

218 aa
Hayashi et al. Figure 2

Identity (\%)

H.Sapiens ys

Protein DNA

$100.0 \quad 100.0$

100.0

91.3

872

88.1

84.5

90.0

84.8

67.6

67.0

Figure 2: LMP2/ $\beta 1$ i genes and proteins conserved in Euteleostomi. 
Citation: Hayashi T, Horiuchi A, Sano K, Gur G, Aburatani H, et al. Proteasome Subunit LMP2/b1i As a Biomarker for Human Uterine Mesenchymal Tumors. J Oncobiomarkers. 2013;1(2): 6.

myometrium located in the same section $[26,27]$. Of the 54 cases examined with human Ut-LMS, 46 were negative for the expression of LMP $2 / \beta 1 \mathrm{i}, 4$ were focally positive, and 2 were partially positive [27] Two Ut-LMS cases stained positive for LMP2/ $\beta 1$ i. LMP $2 / \beta 1$ i levels were also evaluated in the skeletal muscle and rectum metastases from individual Ut-LMS patients [27]. A pathological examination of surgical samples revealed the presence of a mass measuring $3 \mathrm{~cm}$ in diameter in the lumbar quadrate muscle without a fibrous capsule. All lymph nodes were negative for human Ut-LMS metastases, and IHC studies were positive for ki-67 and negative for LMP2/31i [27]. Histological findings were consistent with metastatic Ut-LMS for the skeletal muscle and rectum lesions [27]. Western blotting and RT-PCR experiments revealed that LMP2/ $\beta 1 \mathrm{i}$ was expressed in the normal myometrium, but not in human Ut-LMS, and both these results were strongly supportive of the IHC findings [26,27] (Figure $2 \mathrm{~b})$. To increase the tumor incidence and better assess the role of the systemic expression of TP53 in response to the initiation of Ut-LMS tumorigenesis, LMP2/ $\beta 1$ i-null mice were bred with TP53-null mice to create $L m p 2^{--T p} 53^{--}$double knockout mice. The incidence of UtLMS and death rates were similar in $\operatorname{Lmp} 2^{--} T p 53^{--/}$mice and closely matched those for control $\operatorname{Lmp} 2^{--} T p 53^{+/+}$mice. The correlation between defective TP53 function and Ut-LMS tumorigenesis remains unclear. Although we previously demonstrated that the abnormal expression of ovarian steroid receptors, TP53, ki-67, and mutations of TP53 were frequently associated with human Ut-LMS, defective LMP2/ $\beta 1$ i expression appears to be more characteristic of human UtLMS than any of these factors [26-29].

\section{The biological significance of LMP2/ $\beta 1 i$ in human Ut-LMS tumorigenesis}

The IFN- $\gamma$ pathway is important in the control of tumor growth and invasion and has been implicated in several cancers. Recent studies on human and mouse uterine tissues revealed the defective expression of LMP2/ $\beta 1 \mathrm{i}$ in human Ut-LMS, and this was attributed

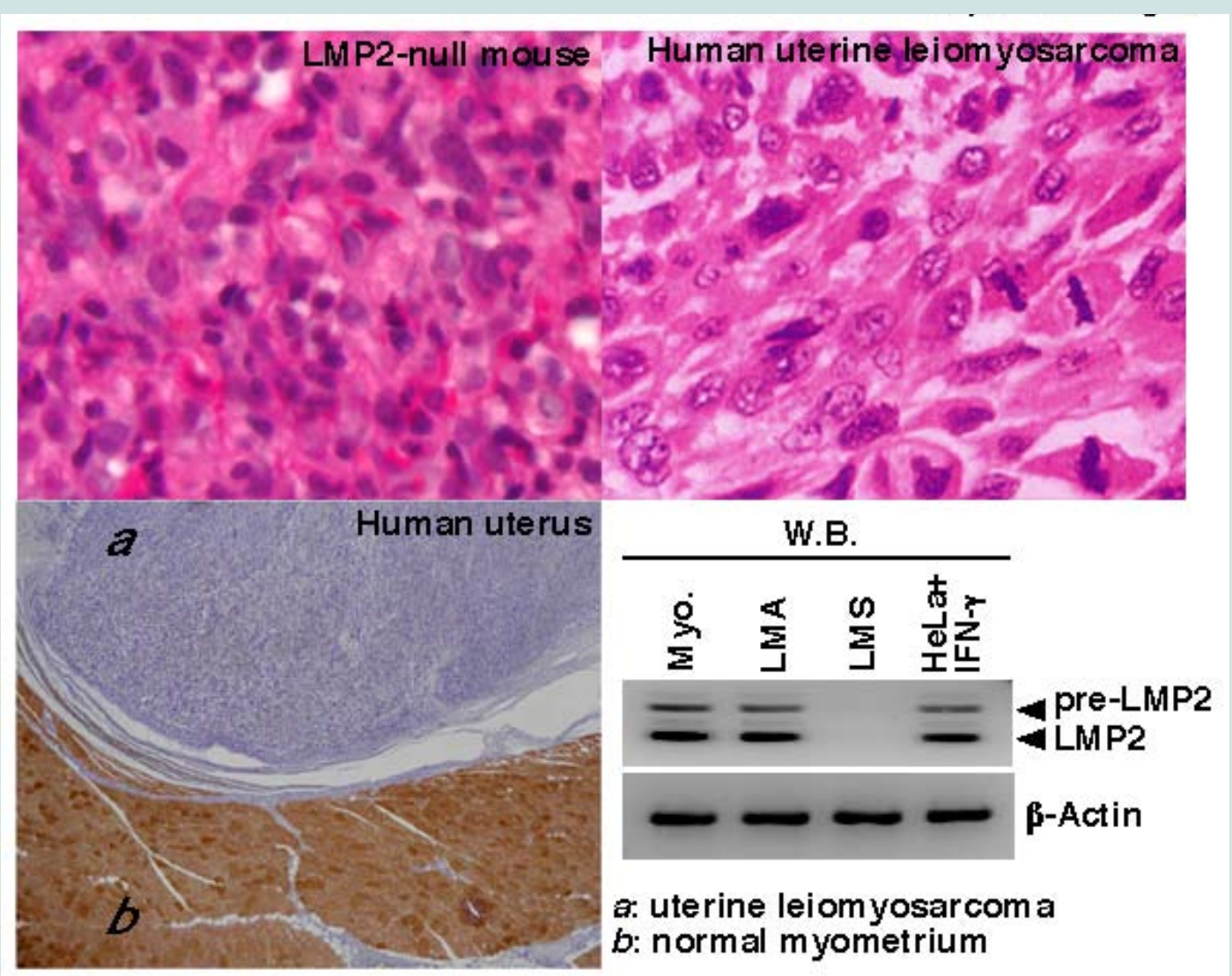

Figure 3: Defective LMP2/ß1i expression in human uterine leiomyosarcoma. H.E. staining tissue sections of uterine leomyosarcoma from a LMP2/B1i-null mouse and patient. Immunohistochemical staining of a tissue section of human uterine leiomyosarcoma with the anti-human LMP2/B1i monoclonal antibody. Extracts of $50 \mu \mathrm{g}$ were resolved by $10 \%$ sodium dodecyl sulfate-polyacrylamide gel electrophoresis (SDS-PAGE). LMP2 and $\beta$-actin levels were examined by immunoblot analysis with appropriate antibodies.

NCBI/ BLAST/ blastp suite-2sequences/ Formatting Results - HP3N9BZD11N

Query ID: gi|4506205|ref|NP 002791.1|

Description: proteasome subunit beta type-9 proprotein [Homo sapiens]

Molecule type: amino acid

Query Length: 219

Subject ID: gi|8567394|ref|NP_038613.1|

Description: proteasome subunit beta type-9 [Mus musculus]

Molecule type: amino acid

Subject Length: 219

Program: BLASTP 2.2.27+ Citation 
Citation: Hayashi T, Horiuchi A, Sano K, Gur G, Aburatani H, et al. Proteasome Subunit LMP2/b1i As a Biomarker for Human Uterine Mesenchymal Tumors. J Oncobiomarkers. 2013;1(2): 6.

to the IFN- $\gamma$ pathway and specific effects of somatic mutations in the catalytic domain of JAK- 1 , an IFN- $\gamma$ receptor-associated tyrosine kinase, on LMP2/ $\beta 1$ i transcriptional activation $[26,27,29]$. This differential responsiveness to the genetically modified stable expression of LMP2/B1i in the SKN human Ut-LMS cell line was investigated to determine whether reintroducing LMP $2 / \beta 1 \mathrm{i}$ would affect tumorigenic properties for the development of human Ut-LMS, and also if the observed effect was due to the immunoproteosomal function of the protein. Recent studies demonstrated that the expression of LMP2/ $\beta 1 \mathrm{i}$ was markedly lower in human Ut-LMS tissues in comparison with uterine LMA and normal myometrium tissues. Thus, LMP2/ $\beta 1$ i may be a potential diagnostic biomarker that can distinguish between Ut-LMS and LMA. Furthermore, we inferred that tumor growth and sarcomagenesis were modulated by LMP2/ $\beta 1$ i expression, implicating LMP $/ \beta 1 \mathrm{i}$ as an anti-oncogenic factor in Ut-LMS. We isolated and expanded the typical clones of each type and analyzed their growth properties, as well as the occurrence and expression of LMP2/ $\beta 1 \mathrm{i}$ and LMP2/ $\beta 1 \mathrm{iK} 33 \mathrm{~A}$, which has no effect on immunoproteasome functions due to its non-incorporation into the 20S proteasome, in these typical clones. Growth rates, indicated as doubling time, were generally lower in the typical SKN-LMP2/B1i (Flatrevertant cell type) clones and SKN-LMP2/ $\beta 1 i K 33 \mathrm{~A}$ (Flatrevertant cell type) clones than in the control SKNCEM9 (Transform cell type) clones. FACS analysis demonstrated that LMP2/ $\beta 1$ i expression might have induced G1 arrest in the SKNLMP2/ $\beta 1$ i (Flatrevertant cell type) clones. The efficiency of colony formation and size of the colonies in soft agar were greatly reduced in the Flatrevertant cell-type clones. Tumor growth was clearly observed in BALB/c nu/nu mice inoculated with SKN-CEM9 (Transform cell type) clones; however, a reduction in tumor growth was noted in mice inoculated with the SKN-LMP2/ $\beta 1$ i (Flatrevertant cell type) clones. A recent study suggested that the suppression of cell proliferation, sarcomagenesis, and morphological changes in uterine LMS cells observed under stable LMP2/ $/$ 1i expression may be attributed to the biological function of single-molecule LMP2/ $\beta 1 \mathrm{i}$ only, without the involvement of its immunoproteasome function. An analysis of human Ut-LMS cells clarified the biological significance of LMP2/ $\beta 1 \mathrm{i}$ in malignant myometrium transformation including the cellular morphological phenotype and cell cycle, thereby implicating LMP2/ $\beta 1 \mathrm{i}$ as an anti-tumorigenic candidate $[26,27,29]$.

In addition, recent studies reported an association between malignant transformation of the myometrium and the reduced expression of calponin h1, a calcium-binding protein, that is specifically expressed in smooth muscle and binds calmodulin, actin, and tropomyosin [29-31]. Calponin h1 reportedly inhibits the ATPase activity of myosin and may play a role in smooth muscle contraction $[32,33]$. Although calponin h1 may function as a tumor suppressor in Ut-LMS, unlike mice lacking LMP2/ß1i, calponin h1null mice do not exhibit Ut-LMS. The biological characterization of human Ut-LMS remains incomplete [29-31]. LMP2/ $/ 1$ i molecules were reportedly associated with cellular factor(s) that regulate cellular processes, such as the cell cycle and gene expression. Cellular biological experiments were performed to examine the biological connection between LMP2/ $\beta 1$ i and calponin h1. The results obtained that LMP2/ $\beta 1$ i-induced cellular morphological phenotypes may be involved in the biological function of calponin h1. Biological experiments on cell morphology following modified-gene expression suggest that single-unit LMP2/ $\beta 1$ 1i-mediated cellular factors other than calponin h1, prevent cell proliferation and sarcomagenicity in
Ut-LMS cells [26,27,29]. Elucidating the mechanism by which LMP2/ $\beta 1$ i-induced biological events, including calponin h1 expression, are regulated may provide a valuable insight into the transformation of cellular phenotypes, control of cell proliferative activity, and the pathogenesis of human uterine mesenchymal tumors at the molecular level. In conclusion, we demonstrated that LMP2/ $\beta 1$ i may be a tumor suppressor in human Ut-LMS. This role of LMP2/ $\beta 1$ i may lead to new therapeutic targets in human Ut-LMS.

\section{Conclusion}

A female hormonal imbalance is often a risk factor for the development of tumors in gynecological cancers, such as breast cancer [15-17]. However, the relationship between the development of human Ut-LMS, a female hormone, and hormone receptors has yet to be clarified in uterine LMA. A recent study showed the expression of Lmp2/ß1i mRNA and protein in luminal and glandular epithelia, placenta villi, trophoblastic shells, and arterial endothelial cells [3436]. These results implicate LMP $2 / \beta 1 \mathrm{i}$ in the invasion of placental villi, degradation of the extracellular matrix, immune tolerance, glandular secretion, and angiogenesis [35]. Further studies may help to elucidate the regulatory role of LMP $2 / \beta 1 \mathrm{i}$ in the implantation of embryos $[28,34,35,37]$. The LMP2/ $\beta 1$ i-null mouse was the first animal model of spontaneous Ut-LMS to be established. The defective expression of LMP2/ $\beta 1$ i may be one of the causes of human UtLMS. The growth of a cell line with normal JAK1 kinase activity was shown to be strongly inhibited by an IFN- $\gamma$ treatment, whereas the growth of JAK1-deficient cell lines was unaffected [38]. The cellular effects of IFN- $\gamma$ include the inhibition of cellular proliferation and influences on apoptosis. Exogenous LMP2/ $\beta 1 \mathrm{i}$ expression resulted in cell growth inhibition in LMP2/ $\beta 1$ i-transfected human Ut-LMS cells, which highly express LMP2/ $\beta 1 i[26,27]$. In contrast the growth of LMP2/ $\beta 1$ i-transfected human Ut-LMS cells was unaffected by the IFN- $\gamma$ treatment $[26,27]$. Taken together, the effect of IFN- $\gamma$ on cell growth inhibition may be attributed to the inducibility of LMP2/ $\beta 1$ i. To demonstrate whether LMP2/ $\beta 1 \mathrm{i}$ is a potential biomarker that can distinguish human Ut-LMS from uterine LMA, we are currently investigating the reliability and characteristics of LMP $2 / \beta 1 \mathrm{i}$ as a diagnostic indicator in several clinical research facilities. This clinical research has yet to be concluded, and large-scale clinical studies need to be performed. The histological and IHC characteristics of uterine mesenchymal tumors including cellular LMA, bizarre LMA, mitotically active LMA, and smooth muscle tumor of uncertain malignant potential (STUMP) are now being examined at our clinical facilities. A clarification of the relationship between these factors and the development of human Ut-LMS, and the identification of specific risk factors may lead to the development of new treatments for this disease. Human Ut-LMS is refractory to chemotherapy and has a poor prognosis. The molecular biological and cytological information obtained from research studies with LMP $2 / \beta 1$ i-null mice will markedly contribute to the development of preventive methods, a potential diagnostic biomarker, and new therapeutic approaches against human Ut-LMS.

\section{References}

1. Peters JM, Franke WW, Kleinschmidt JA (1994) Distinct $19 \mathrm{~S}$ and $20 \mathrm{~S}$ subcomplexes of the $26 \mathrm{~S}$ proteasome and their distribution in the nucleus and the cytoplasm. J Biol Chem 269: 7709-7718.

2. Voges D, Zwickl P, Baumeister W (1999) The 26S proteasome: A molecular machine designed for controlled proteolysis. Annu Rev Biochem 68: 10151068. 
Citation: Hayashi T, Horiuchi A, Sano K, Gur G, Aburatani H, et al. Proteasome Subunit LMP2/b1i As a Biomarker for Human Uterine Mesenchymal Tumors. J Oncobiomarkers. 2013;1(2): 6.

3. Smith DM, Chang SC, Park S, Finley D, Cheng Y, Goldberg AL (2007) Docking of the proteasomal ATPases' carboxyl termini in the 20S proteasome's alpha ring opens the gate for substrate entry. Mol Cell 27: 731-744.

4. Wolf DH, Hilt W (2004) The proteasome: a proteolytic nanomachine of cell regulation and waste disposal. Biochim Biophys Acta 1695: 19-31.

5. Hayashi T, Faustman DL (2002) Development of spontaneous uterine tumors in low molecular mass polypeptide-2 knockout mice. Cancer Res 62: 24-27.

6. Susumu Tonegawa, proteasome (prosome, macropain) subunit, beta type 9 (large multifunctional peptidase 2); targeted mutation 1.

7. Zaloudek C, Hendrickson MR (2002) Mesenchymal tumors of the uterus, in Kurman RJ (edn): Blaustein's Pathology of the Female Genital Tract (5thedn), New York, Springer-Verlag, 5: 561-578

8. Perez EA, Pusztai L, Van de VM (2004) Improving patient care through molecular diagnostics. Semin Oncol 31: 14-20.

9. Leitao MM, Soslow RA, Nonaka D, AOlshen AB, Aghajanian C. et al (2004) Tissue microarray immuno-histochemical expression of estrogen, progesterone, and androgen receptors in uterine leiomyomata and leiomyosarcoma. Cancer 101: 1455-1462.

10. Wu TT, Chang TC, Hsueh, S Hsu KH, Chou HH, et al. (2006) Prognostic factors and impact of adjuvant chemotherapy for uterine leiomyosarcoma. Gynecol Oncol 100: 166-172.

11. General Information About Uterine Sarcoma

12. Evans HL, Chawla SP, Simpson C, Finn KP (1988) Smooth muscle neoplasms of the uterus other than ordinary leiomyoma. A study of 46 cases, with emphasis on diagnostic criteria and prognostic factors. Cancer 62: 2239

13. Kurma RJ. Pathology of the Female Genital Tract (4thedn) New York, Springer-Verlag 4: 499

14. (2003) Diagnostic Criteria for LMS, Adapted from 2003 WHO Guidelines: World Health Organization Classification of Tumours: Pathology and Genetics of Tumours of the Breast and Female Genital Organs; IARC Press, France..

15. Miettinen M, Fetsch JF (2006) Evaluation of biological potential of smooth muscle tumours. Histopathol 48: 97-105.

16. Lin JF, Slomovitz BM (2008) Uterine sarcoma. Current Oncol Report 10: 512 518.

17. Amant F, Coosemans A, Debiec-Rychter M, Timmerman D, Vergote I (2009) Clinical management of uterine sarcomas. Lancet Oncol 10: 1188-1198.

18. Peters JM, Franke WW, Kleinschmidt JA (1994) Distinct $19 S$ and 20 S subcomplexes of the $26 \mathrm{~S}$ proteasome and their distribution in the nucleus and the cytoplasm. J Biol Chem 269: 7709-7718.

19. Lodish H, Berk A, Matsudaira P, Kaiser CA, Krieger M, et al. (2004) Molecula Cell Biology (5thedn), New York: W.H. Freeman and CO. 5: 66-72.

20. Higashitsuji H, Liu Y, Mayer RJ, Fujita J (2005) The oncoprotein gankyrin negatively regulates both $\mathrm{p} 53$ and RB by enhancing proteasomal degradation. Cell Cycle 4: 1335-1337.

21. Konstantinova IM, Tsimokha AS, Mittenberg AG (2008) Role of proteasomes in cellular regulation. Intl Rev Cell Mol Biol 267: 59-124.

22. Wang J, Maldonado MA (2006) The Ubiquitin-Proteasome System and Its
Role in Inflammatory and Autoimmune Diseases. Cell Mol Immunol 3: 255261

23. Muchamuel T, Basler M, Aujay MA, Suzuki E, Kalim KW et al. (2009) A selective inhibitor of the immunoproteasome subunit LMP7 blocks cytokine production and attenuates progression of experimental arthritis. Nature Med 15: 781-788.

24. Van Kaer L, Ashton-Rickardt A, Eichelberger PG, Gaczynska M, Nagashima M, et al. (1994) Altered peptidase and viral-specific T cell response in LMP2 mutant mice. Immunity 1: 533-541.

25. Hayashi T, Horiuchi A, Sano K, Hiraoka N, Kanai Y, et al. (2011) Molecular approach on uterine leiomyosarcoma: LMP2-deficient mice as an animal model of spontaneous uterine leiomyosarcoma. Sarcoma 2011: 476-498.

26. Hayashi T, Kobayashi Y, Kohsaka S, Sano K (2006) The mutation in the ATPbinding region of JAK1, identified in human uterine leiomyosarcomas, results in defective interferon-gamma inducibility of TAP1 and LMP2. Oncogene 25 4016-4026.

27. Hayashi T, Horiuchi A, Sano K, Hiraoka N, Kasai M, et al. (2011) Potential role of LMP2 as tumor-suppressor defines new targets for uterine leiomyosarcoma therapy. Sci Rep.

28. Zhai YI, Kobayashi Y, Mori A, Orii A, Nikaido T, et al. (1999) Expression of steroid receptors, Ki-67, and p53 in uterine leiomyosarcomas. Intl J Gynecol Pathol 18: 20-28.

29. Hayashi T, Horiuchi A, Sano K, Hiraoka N, Kasai M, et al. (2012) Potential role of LMP2 as an anti-oncogenic factor in human uterine leiomyosarcoma morphological significance of calponin h1. FEBS Lett 586: 1824-1831.

30. Horiuchi A, Nikaido T, Ito K, Zhai YI, Orii A, et al. (1998) Reduced expression of calponin h1 in leiomyosarcoma of the uterus. Lab Invest 78: 839-846.

31. Horiuchi A, Nikaido T, Taniguchi S, Fujii S (1999) Possible role of calponin h1 as a tumor suppressor in human uterine leiomyosarcoma. J Natl Can Inst 91: 790-796

32. Winder SJ, Walsh MP, Vasulka C, Johnson JD (1993) Calponin-calmodulin interaction: properties and effects on smooth and skeletal muscle actin biding and actomyosin ATPases. Biochemistry 32: 13327-13333.

33. Wills FL, McCubbin WD, Kay CM (1994) Smooth muscle calponin-caltropin interaction: effect on biological activity and stability of calponin. Biochem 33 $5562-5569$

34. Wang HX, Wang HM, Li QL, Judoson PL (2004) Expression of proteasome subunits low molecular mass polypeptide (LMP) 2 and LMP7 in the endometrium and placenta of rhesus monkey (Macaca mulatta) during early pregnancy. Biol Repro 71: 1317-1324.

35. Wang $\mathrm{HX}$, Wang HM, Lin HY, Yang Q, Zhang $\mathrm{H}$ (2006) Proteasome subunit LMP2 is required for matrix metalloproteinase-2 and -9 expression and activities in human invasive extravillous trophoblast cell line. J Cell Physio 206: 616-623.35.

36. Fu JJ, Lin P, Lv XY, Yan XJ, Wang HX, et al. (2009) Low molecular mass polypeptide-2 in human trophoblast: over-expression in hydatidiform moles and possible role in trophoblast cell invasion. Placenta 30: 305-312.

37. Sexl V, Kovacic B, Piekorz R, Moriggl R, Stoiber D, et al. (2003) Jak1 deficiency leads to enhanced Abelson-induced B-cell tumor formation, Blood 101: 4937-4943.

\section{Acknowledgements}

We sincerely thank Professor Luc Van Kaer (Vanderbilt University Medical Center). This study was supported in part by grants from the Ministry of Education, Culture, Science and Technology, and The Foundation of Osaka Cancer Research, The Ichiro Kanehara Foundation for the Promotion of Medical Science and Medical Care, The foundation for the Promotion of Cancer Research, The Kanzawa Medical Research Foundation, The Shinshu Medical Foundation, and The Takeda Foundation for Medical Science. 